\title{
Molecular dynamics with constrained nuclear electronic orbital density functional theory: Accurate vibrational spectra from efficient incorporation of nuclear quantum effects
}

\author{
Xi Xu, Zehua Chen, and Yang Yang* \\ Theoretical Chemistry Institute and Department of Chemistry, University of \\ Wisconsin-Madison, 1101 University Avenue, Madison, WI 53706, USA \\ E-mail: yyang222@wisc.edu
}

\begin{abstract}
Nuclear quantum effects play a crucial role in many chemical and biological systems involving hydrogen atoms yet are difficult to include in practical molecular simulations. In this Letter, we combine our recently developed methods of constrained nuclearelectronic orbital density functional theory (cNEO-DFT) and constrained minimized energy surface molecular dynamics (CMES-MD) to create a new method for accurately and efficiently describing nuclear quantum effects in molecular simulations. Using this new method, dubbed cNEO-MD, the vibrational spectra of a set of small molecules are calculated and compared with those from conventional ab initio molecular dynamics (AIMD) as well as from experiments. With the same formal scaling, cNEO-MD greatly outperforms AIMD in describing the vibrational modes with significant hydrogen motion characters, demonstrating the promise of cNEO-MD for simulating chemical and biological systems with significant nuclear quantum effects.
\end{abstract}


Molecular dynamics (MD) simulations are a powerful tool for investigating static and dynamic properties of chemical and biological systems. ${ }^{1-3}$ In atomistic MD simulations, atoms evolve on potential energy surfaces (PESs) according to Newtonian dynamics, and thus an accurate PES is necessary for a reliable simulation. In general, PESs can be obtained via either empirical force fields or $a b$ initio electronic structure calculations. MD simulations based on force fields are computationally efficient, whereas ab initio molecular dynamics (AIMD), which uses PESs calculated on-the-fly by ab initio electronic structure calculations, is generally more accurate, especially in describing electronic polarizations and chemical reactions. However, in conventional AIMD simulations, all nuclei evolve classically on a PES that does not include nuclear quantum effects such as zero-point energies and quantum tunneling. As a result, AIMD can perform poorly in the description of many important chemical and biological systems involving hydrogen atoms, such as water, in which nuclear quantum effects play an important role in its hydrogen bonding network. 4 - 1 ]

Many methods have been developed to incorporate nuclear quantum effects in molecular simulations. $\frac{8[10}{[10}$ Wave packet dynamics $\frac{9111}{}$ can be very accurate, but the cost scales up rapidly with the system size and is usually limited to systems with tens of degrees of freedom. Methods based on the path-integral formalism can also describe nuclear quantum effects and are more popular in practical applications. They are based on a classical-quantum isomorphism, 12113 which maps the underlying quantum system onto a classical one with chains of replicas. In this way, static properties can be obtained by the ensemble average within the extended phase space with either molecular dynamics (PIMD) or Monte Carlo (PIMC) simulations, and dynamical properties can be obtained with centroid molecular dynamics ${ }^{14}$ (CMD), ring-polymer molecular dynamics ${ }^{15}$ (RPMD), and other variants. ${ }^{16117}$ These pathintegral methods have been applied to a variety of aqueous and solid systems and have provided many important chemical insights. ${ }^{18119}$ However, while static properties can mostly be accurately described, dynamical properties such as vibrational spectra remain challenging: RPMD can have spurious peak splittings due to the unphysical resonance between the 
molecular vibrational modes and the internal vibrational modes of the ring polymer, 20121 while CMD can suffer from an artificial redshift for stretching modes due to the curvature problem at low temperatures. $\frac{22}{2}$ Furthermore, although techniques have been developed to accelerate PIMD simulations, 10 very few of them can be directly extended to RPMD and CMD simulations, and it remains computationally expensive to perform RPMD and CMD simulations on molecular systems with accurate $a b$ initio PESs. Therefore, a molecular simulation method that can accurately and efficiently incorporate nuclear quantum effects remains highly desirable.

Recently, our group developed the constrained nuclear-electronic orbital density functional theory 2324 (cNEO-DFT) as an extension to the multicomponent density functional theory. 25 A significant advantage of cNEO-DFT is that it yields an energy surface that naturally incorporates nuclear quantum effects. This surface is a function of quantum nuclear expectation positions as well as classical nuclear positions of any nuclei treated classically. With cNEO-DFT energy surfaces, accurate vibrational frequencies have been obtained for a series of small molecules with harmonic Hessian calculations, $\frac{31}{21}$ which are significantly more accurate than those from conventional DFT calculations and are comparable to or even better than those from the vibrational perturbation theory (DFT-VPT2). 32 These results suggest that cNEO-DFT gives reliable energy surfaces, at least around local energy minima, and thus is promising for performing dynamics. Furthermore, our group also recently developed constrained minimized energy surface molecular dynamics (CMES-MD), which modifies classical molecular dynamics to incorporate nuclear quantum effects. In this Letter, CMES-MD simulations are performed in molecular systems with cNEO-DFT energy surfaces, and this new approach, dubbed cNEO-MD, is employed to calculate the vibrational spectra of a set of small molecules. The results are compared with those from AIMD as well as from experiments to demonstrate the promise of cNEO-MD for molecular simulations.

In multicomponent DFT, the ground-state energy of a molecular system is expressed as a functional of electronic densities and quantum nuclear densities, 25 


$$
E\left[\rho_{e},\left\{\rho_{n}\right\}\right],
$$

where $\rho_{e}$ denotes the electron density and $\rho_{n}$ denotes the density of the $n$ th-type quantum nucleus. As with conventional electronic DFT, in principle, the ground state energy as well as the ground state electronic and nuclear densities can be obtained if the exact density functional is known. However, in practice, approximations are needed, and much progress has been made in developing multicomponent density functional approximations to obtain accurate ground-state properties $\frac{35136}{36}$

As an extension to multicomponent DFT, cNEO-DFT is developed from the fact that in regular chemical and biological systems, quantum nuclei are generally localized in space, and therefore can be treated as distinguishable particles with certain nuclear expectation positions, which can be expressed as:

$$
\left\langle\mathbf{r}_{I}\right\rangle \equiv \int \mathbf{r} \rho_{I}(\mathbf{r}) \mathrm{d} \mathbf{r}=\mathbf{R}_{I},
$$

where $\left\langle\mathbf{r}_{I}\right\rangle$ denotes the expectation position for the $I$ th quantum nucleus and $\mathbf{R}_{I}$ is the value of the expectation position. Thus, the quantum state for a set of defined quantum nuclear expectation positions can be obtained via a constrained energy minimization with the following additional terms in the Lagrangian 2324

$$
\sum_{I} \mathbf{f}_{I} \cdot\left(\left\langle\mathbf{r}_{I}\right\rangle-\mathbf{R}_{I}\right),
$$

where $\mathbf{f}_{I}$ is the Lagrange multiplier associated with the Ith quantum nucleus.

Making the Lagrangian stationary with respect to electronic and nuclear densities leads to a set of coupled Fock equations for electrons and quantum nuclei, which need to be solved self-consistently together with the Lagrange multipliers $\left\{\mathbf{f}_{I}\right\}$. The resulting cNEO-DFT is a constrained minimized energy surface (CMES) and is a function of quantum nuclear expec- 
tation positions as well as classical nuclear positions if certain nuclei are treated classically. The analytic energy gradients ${ }^{24}$ can be directly calculated by taking derivatives with respect to the expectation positions of quantum nuclei and the coordinates of classical nuclei, which will serve as the forces acting on the nuclei in subsequent MD simulations.

Our group has recently developed constrained minimized energy surface molecular dynamics (CMES-MD), which is a general theory for including nuclear quantum effects in molecular dynamics simulations. CMES-MD has been shown to accurately describe the quantum harmonic oscillator, the Morse oscillator, and a double-well potential, demonstrating the promise of CMES-MD in accurately and efficiently incorporating nuclear quantum effects, especially zero-point effects and tunneling effects. $\stackrel{37}{2}$ In CMES-MD, the equations of motion are

$$
m \frac{\mathrm{d}\langle\mathbf{x}\rangle}{\mathrm{d} t}=\langle\mathbf{p}\rangle
$$

and

$$
\frac{\mathrm{d}\langle\mathbf{p}\rangle}{\mathrm{d} t} \approx-\frac{\mathrm{d} V^{\text {CMES }}}{\mathrm{d}\langle\mathbf{x}\rangle},
$$

in which $V^{\mathrm{CMES}}$ is the constrained minimized energy surface with the quantum particle constrained at the expectation position $\langle\mathbf{x}\rangle$. These equations are highly similar to those of classical molecular dynamics with the difference that $V^{\mathrm{CMES}}$ is an effective potential that incorporates nuclear quantum effects, replacing the conventional potential. In practical molecular systems, since cNEO-DFT minimizes the energy with constrained nuclear positions, the cNEO-DFT energy surface is naturally the CMES, and thus may be utilized for CMES-MD simulations, which we now call cNEO-MD. With highly similar equations of motion, cNEO-MD can be performed essentially in the same way as conventional AIMD, with multicomponent cNEO-DFT calculations replacing the pure electronic structure calculations of AIMD for obtaining energies and gradients. As the computational scalings of cNEO-DFT 
and conventional DFT are the same, cNEO-MD is only more expensive than conventional AIMD by a prefactor, which depends on the number of quantum nuclei.

We use cNEO-MD to compute the vibrational spectra of three molecular systems-water $\left(\mathrm{H}_{2} \mathrm{O}, \mathrm{HDO}, \mathrm{D}_{2} \mathrm{O}\right)$, formic acid $(\mathrm{HCOOH})$, and methanol $\left(\mathrm{CH}_{3} \mathrm{OH}\right)$. During the MD simulations, the cNEO-DFT energy and gradient calculations are performed on-the-fly with an in-house version of PySCF, $\stackrel{38139}{ }$ and the MD simulations are performed with the Atomic Simulation Environment (ASE) package. ${ }^{40}$ For cNEO-DFT calculations, since nuclear quantum effects are most significant for hydrogen atoms, we only treat hydrogen atoms quantum

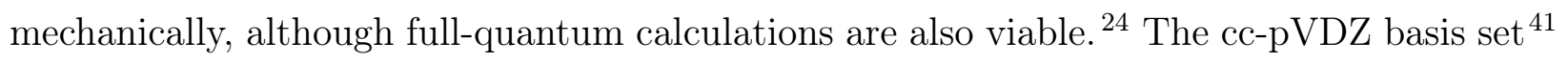
is adopted for electrons and the PB4-D basis ${ }^{42}$ is used for protons. A modified PB4-D basis is used for deuterium with exponents rescaled according to the mass dependence in the harmonic oscillator model. (Table S1) In order to minimize the error introduced by the electronic exchange-correlation functional, we benchmarked and adopted the PBE0 functional ${ }^{43}$ because it gave the most accurate results for molecular vibrational frequencies when compared to $\operatorname{CCSD}(\mathrm{T}) . \underline{44}$ (see Table S2 for benchmark details) We do not include electron-nuclei correlations or nuclei-nuclei correlations, and their influence will be left for future studies. In MD simulations, an NVT simulation is first performed to sample the canonical equilibrium distribution with the Nose-Hoover thermostat. $\stackrel{45[46}{~ T h e ~ t i m e ~ s t e p ~ i s ~ s e t ~ t o ~} 0.5$ fs and the total simulation time is 20 ps. After reaching equilibrium, five uncoupled phase-space points are picked as the starting points for five independent $N V E$ simulations with a time step of 0.5 fs and a total simulation time of 5 ps. Based on the $N V E$ trajectories, velocity autocorrelation functions and dipole autocorrelation functions are then calculated and used to obtain power spectra and infrared (IR) spectra, respectively, through a Fourier transform and trajectory averaging. $\underline{47-49}$ (see Figs. S1, S2 and S3 for the results of different trajectories) For comparison, classical AIMD simulations based on DFT-PBE0 PESs are performed with the same parameters.

The IR spectra of single $\mathrm{H}_{2} \mathrm{O}$, HDO and $\mathrm{D}_{2} \mathrm{O}$ molecules by cNEO-MD and AIMD at 
$300 \mathrm{~K}$ are shown in Fig. 1 along with the experimental vibrational frequencies indicated by dashed vertical lines. There are three vibrational modes in these molecules, all of which are IR active. It has been widely known that AIMD tends to overestimate the vibrational frequencies of water molecules, particularly for $\mathrm{O}-\mathrm{H}(\mathrm{D})$ stretch modes. The mean absolute error (MAE) of AIMD simulations for these peaks is $95 \mathrm{~cm}^{-1}$. In contrast, the vibrational frequencies by cNEO-MD are significantly more accurate with a MAE of $17 \mathrm{~cm}^{-1}$. Hence, excellent results are calculated from cNEO-MD regardless of the isotope type. When compared with the corresponding harmonic Hessian results, both AIMD and cNEO-MD lower the harmonic frequencies by only about $10 \mathrm{~cm}^{-1}$ (See Table S3 for details). In contrast, the differences between AIMD and cNEO-MD frequencies are roughly $100 \mathrm{~cm}^{-1}$. Therefore, we may conclude that nuclear quantum effects are more important than temperature-mediated anharmonicity in the accurate description of vibrational frequencies for water molecules, although we note that nuclear quantum effects and anharmonic effects are often closely related. .50 In addition to peak positions, the intensities of the peaks are also reasonable with a higher intensity for the asymmetric O-H(D) stretch than for the symmetric O-H(D) stretch.

It is known that there exists a Fermi resonance between the fundamental of the O-D stretch mode and the first overtone of the bend mode in the HDO molecule ${ }^{\sqrt{51 / 52}}$ as a result of their proximity in frequency. This Fermi resonance is manifested by two peaks close in both position and intensity, and this subtle feature has been captured by cNEO-MD with a doublet at around $2710 \mathrm{~cm}^{-1}$ and $2740 \mathrm{~cm}^{-1}$ (see inset of Fig. 1 and Fig. S1(b)). In contrast, AIMD fails to describe the Fermi resonance with only a single peak at $2806 \mathrm{~cm}^{-1}$. This difference in performance suggests that cNEO-MD also greatly outperforms AIMD in describing overtones.

Previously, the vibrational spectrum of a single $\mathrm{H}_{2} \mathrm{O}$ molecule has been calculated with RPMD,$[53$ a popular method for including nuclear quantum effects. According to this study, RPMD yields vibrational frequencies that are about $100 \mathrm{~cm}^{-1}$ lower than those by harmonic calculations. ${ }^{53}$ However, they are still about $100 \mathrm{~cm}^{-1}$ higher than experimental values. .53 
Furthermore, as the computational cost of cNEO-MD is much smaller than RPMD, cNEOMD is both more accurate and more efficient in predicting vibrational frequencies of the single water molecule. This is consistent with our previous results in a Morse oscillator model, where CMES-MD is more accurate than RPMD and CMD ${ }^{37}$ However, we note that many variants of RPMD and CMD have been recently developed to overcome these limitations, 17754 and comparisons between cNEO-MD and these variants are left for future research.

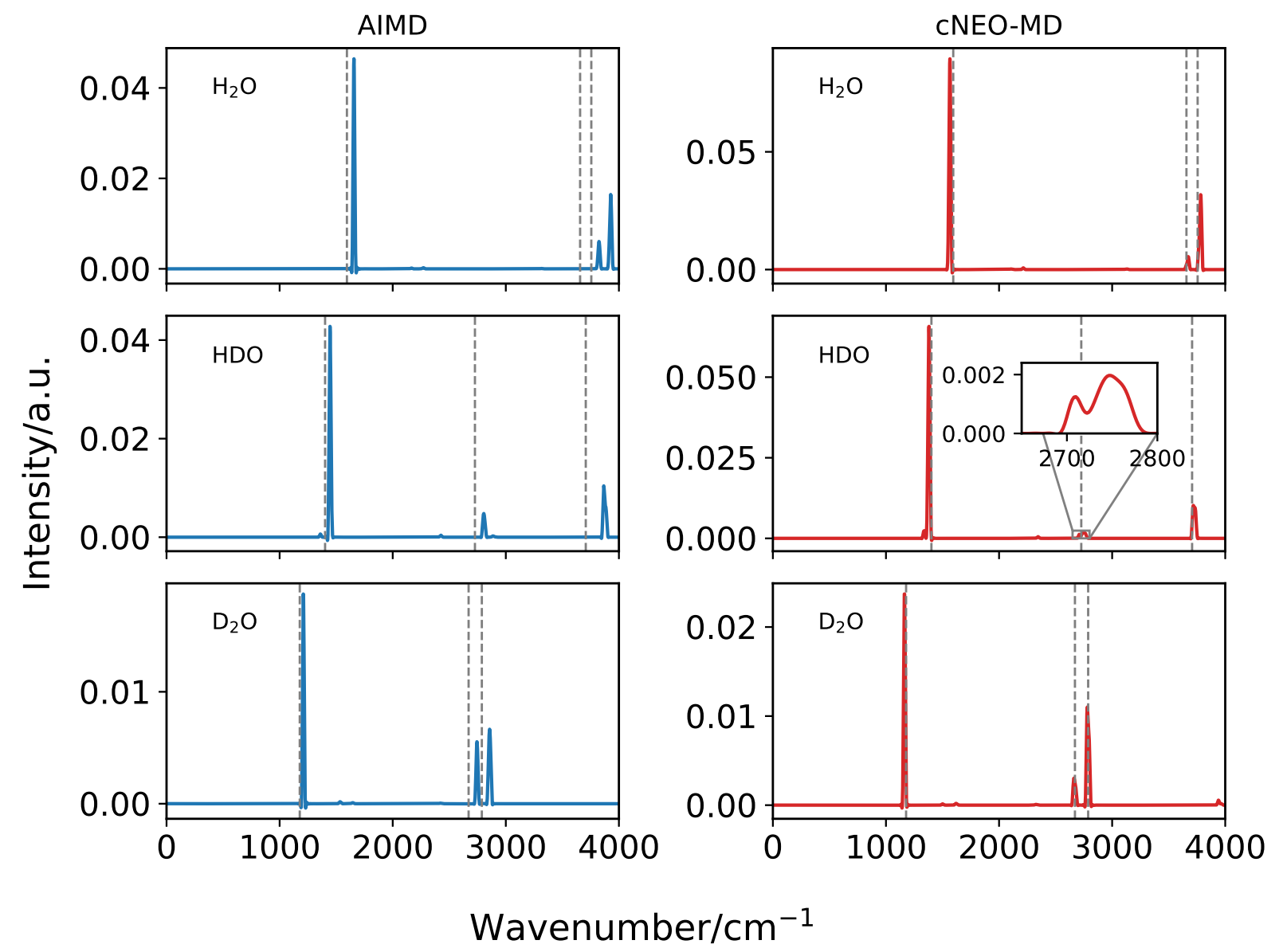

Figure 1: IR spectra of single $\mathrm{H}_{2} \mathrm{O}$, HDO and $\mathrm{D}_{2} \mathrm{O}$ molecules by AIMD and cNEO-MD at $300 \mathrm{~K}$. Experimental vibrational frequencies are shown as dashed vertical lines. The inset for HDO shows the Fermi resonance between the O-D asymmetric stretch mode and the first overtone of the bend mode in a HDO molecule.

The power and IR spectra of a single $\mathrm{HCOOH}$ molecule are presented in Fig. 2a and 2b, respectively. The experimental vibrational frequencies are shown as dashed lines in Fig. 2a, 
Similar to the results for water, AIMD significantly overestimates the frequencies of the two high-frequency C-H stretch and $\mathrm{O}-\mathrm{H}$ stretch modes at around 3000 and $3500 \mathrm{~cm}^{-1}$, by more than $100 \mathrm{~cm}^{-1}$, whereas cNEO-MD can describe them accurately with errors less than 15 $\mathrm{cm}^{-1}$. Hence, cNEO-DFT continues to give substantially improved vibrational frequencies relative to AIMD for vibrations with substantial hydrogen motion character. Nevertheless, for the $\mathrm{C}=\mathrm{O}$ stretch mode near $1700 \mathrm{~cm}^{-1}$, both AIMD and cNEO-MD overestimate its frequency by about $100 \mathrm{~cm}^{-1}$. This is partially caused by the classical treatment of $\mathrm{C}$ and $\mathrm{O}$ atoms in cNEO-DFT calculations, but it is in fact mainly due to the poor performance of PBE0 in describing this stretch mode, which overestimates the frequency by $72 \mathrm{~cm}^{-1}$ compared to $\operatorname{CCSD}(\mathrm{T})$ in a harmonic treatment. (see Table S2) In principle, with a better electronic functional that matches the $\operatorname{CCSD}(\mathrm{T})$ results, this mode should be able to be better described by both AIMD and cNEO-MD.

A Fermi resonance in $\mathrm{HCOOH}$ has been observed experimentally at 1216 and $1306 \mathrm{~cm}^{-1}$, which was attributed to the resonance between the fundamental of the $\mathrm{O}-\mathrm{H}$ bend and the first overtone of the $\mathrm{COH}$ torsion. $\frac{55}{5}$ There is also a doublet in the AIMD results (1378 and $1405 \mathrm{~cm}^{-1}$ ) in Fig. 2a. However, the doublet in AIMD is caused by the Fermi resonance between the $\mathrm{C}-\mathrm{H}$ bend and the overtone of the torsion, while for cNEO-MD, there are three peaks close in energy and intensity, which involve the fundamentals of the $\mathrm{O}-\mathrm{H}$ bend and the $\mathrm{C}-\mathrm{H}$ bend, as well as the overtone of the torsion. The reason for the contaminations from the $\mathrm{C}-\mathrm{H}$ bend in both AIMD and cNEO-MD is again related to the performance of PBE0, which is inaccurate in describing the torsion mode. The frequency of the torsion mode by DFT-PBE0 is overestimated by about $70 \mathrm{~cm}^{-1}$ relative to the experimental value (see Table S3), making the frequency of its overtone too high and predicting a Fermi resonance with both the C-H bend and the $\mathrm{O}-\mathrm{H}$ bend at 1336 and $1385 \mathrm{~cm}^{-1}$ in the cNEO-MD simulation. We note that although PBE0 is problematic in describing some of the modes in $\mathrm{HCOOH}$, it is still the most accurate of all the functionals we tested (Table S2). This calls for more density functional development in the accurate description of molecular vibrations. 
For the IR spectra in Fig. 2b, cNEO-MD produces a spectrum that is in great agreement with the experimental results for both peak positions and peak intensities. A major difference is that the experimental results have peak splittings due to molecular rotations whereas rotations are removed from all our MD simulations. As with the power spectrum, AIMD is less accurate than cNEO-MD since it greatly overestimates the frequencies of the $\mathrm{C}-\mathrm{H}$ and O-H stretch modes.

The power and IR spectra of $\mathrm{CH}_{3} \mathrm{OH}$ by AIMD and cNEO-MD at $300 \mathrm{~K}$ are provided in Fig. 3 . The experimental vibrational frequencies are shown as dashed lines in Fig. 3a, Similar to the previous cases, the $\mathrm{O}-\mathrm{H}$ stretch mode at $3681 \mathrm{~cm}^{-1}$ is accurately predicted by cNEO-MD with an error of $6 \mathrm{~cm}^{-1}$, whereas AIMD gives a significant overestimation of the frequency with an error of $145 \mathrm{~cm}^{-1}$. There are three experimental reference values in the range of 2800 to $3000 \mathrm{~cm}^{-1}$ that are associated with $\mathrm{CH}_{3}$ stretches. However, we can observe four peaks in this range for both AIMD and cNEO-MD due to the Fermi resonances between the $\mathrm{CH}_{3}$ stretch modes and the overtone of the $\mathrm{CH}_{3}$ deformation modes, which is observed experimentally in the Raman spectrum of gaseous methanol. 56 The frequency range for these peaks by cNEO-MD is closer to the experimental result than that by AIMD, which can also be verified by the good agreement between the IR spectrum by cNEO-MD and the experimental spectrum in Fig. 3b. Similar behaviors can be observed for the $\mathrm{CH}_{3}$ deformation modes at around $1500 \mathrm{~cm}^{-1}$ and $\mathrm{CH}_{3}$ rock modes at around $1000 \mathrm{~cm}^{-1}$, all of which suggest that cNEO-MD produces an IR spectrum that more accurately matches the experimental result than AIMD.

In MD simulations, it is well known that the simulation temperature can affect the appearance of spectra. In principle, the temperature dependence study needs to be performed through an ensemble average of a series of $N V E$ simulations for any particular temperature $T$. However, this requires a large number of simulations and makes it hard to converge the final spectra, particularly at high temperatures. Following a similar treatment in Refs. [57,58, we directly used the spectra from a single $N V T$ trajectory to investigate the temperature 


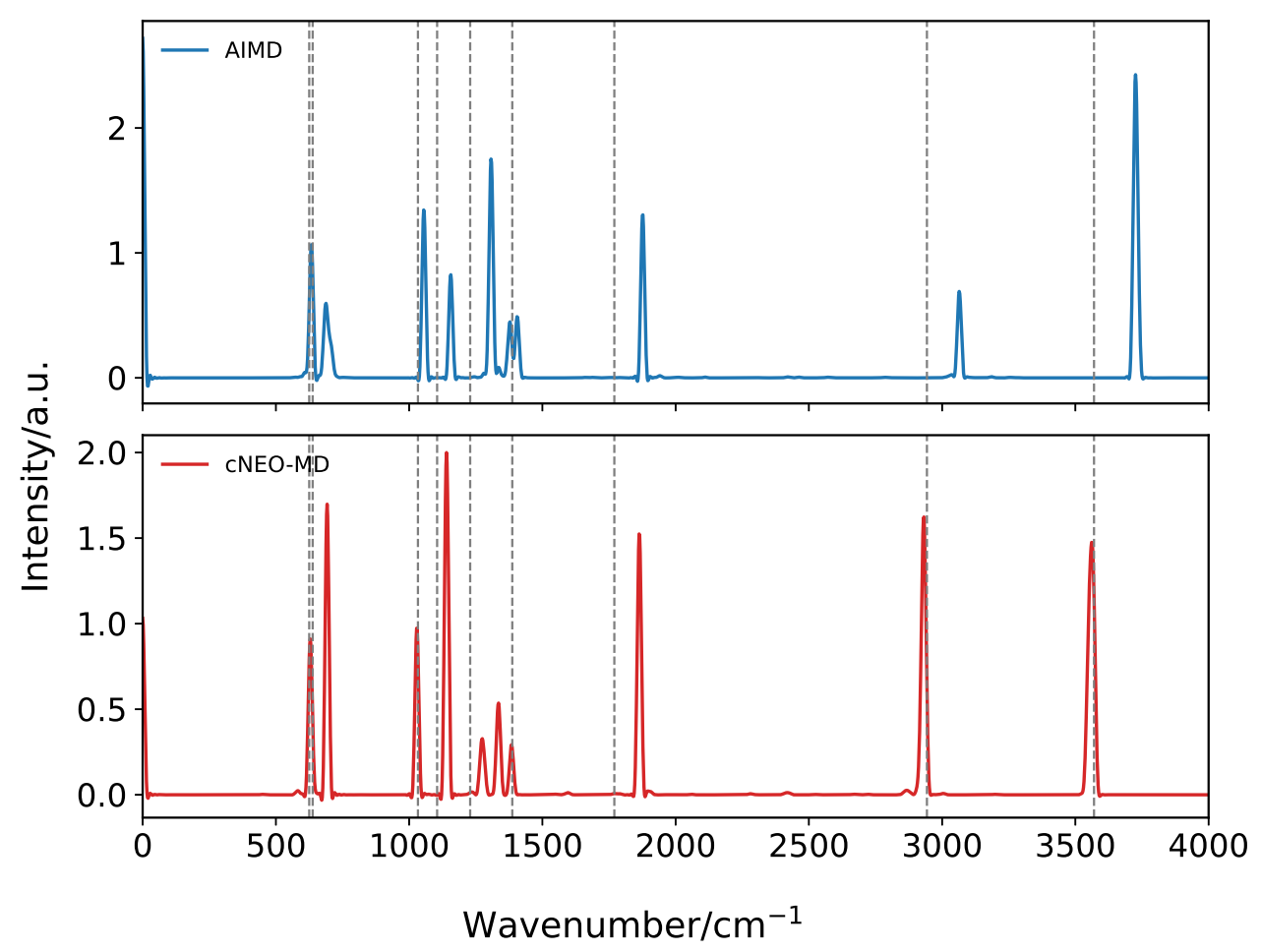

(a) Power spectra
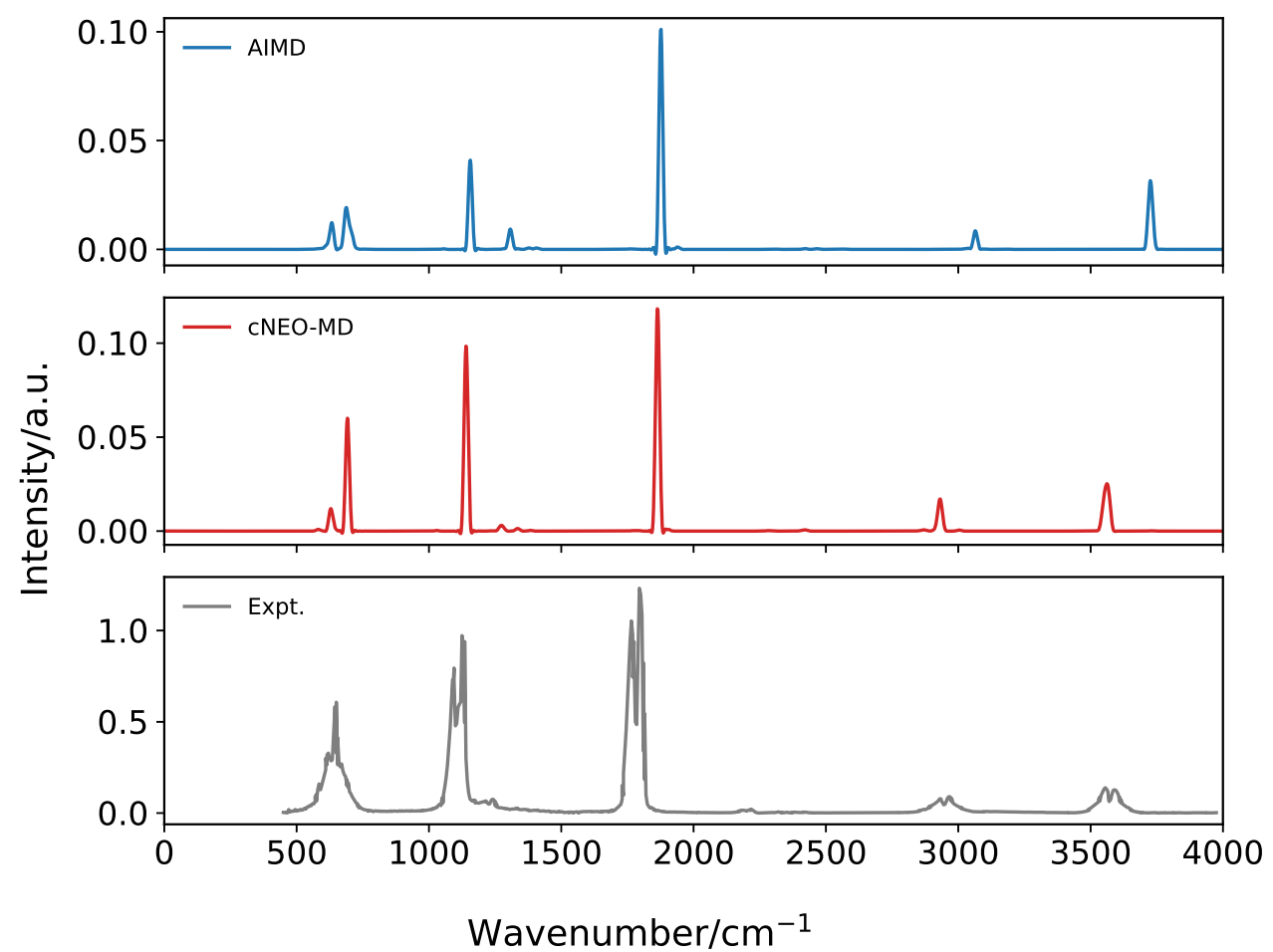

(b) IR spectra

Figure 2: Power spectra and IR spectra of a single $\mathrm{HCOOH}$ molecule by AIMD and cNEO$\mathrm{MD}$ at $300 \mathrm{~K}$. Experimental vibrational frequencies shown as dashed vertical lines in (a) and experimental spectra in (b) are both from National Institute of Standards and Technology (NIST) websites. 


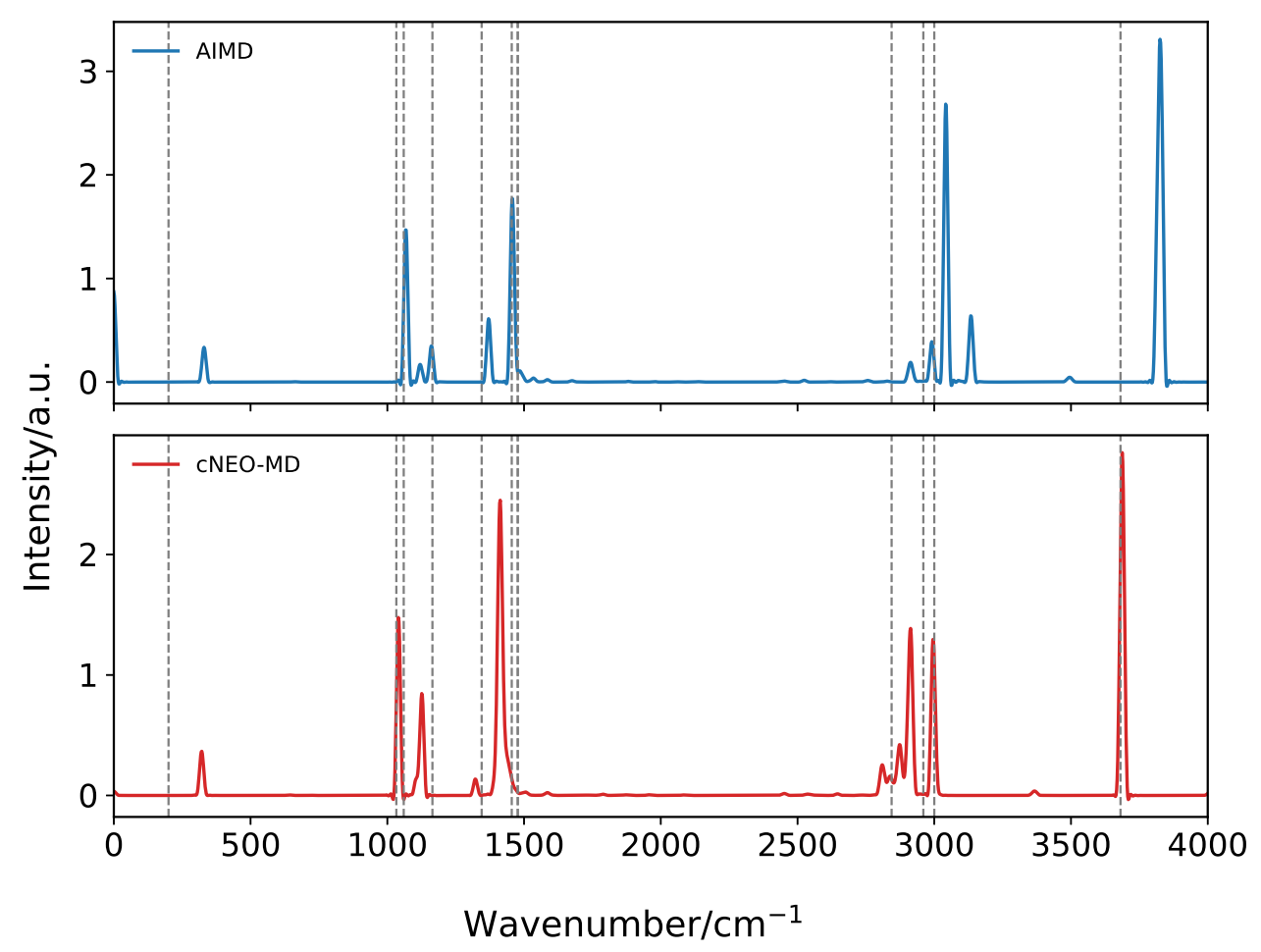

(a) Power spectra
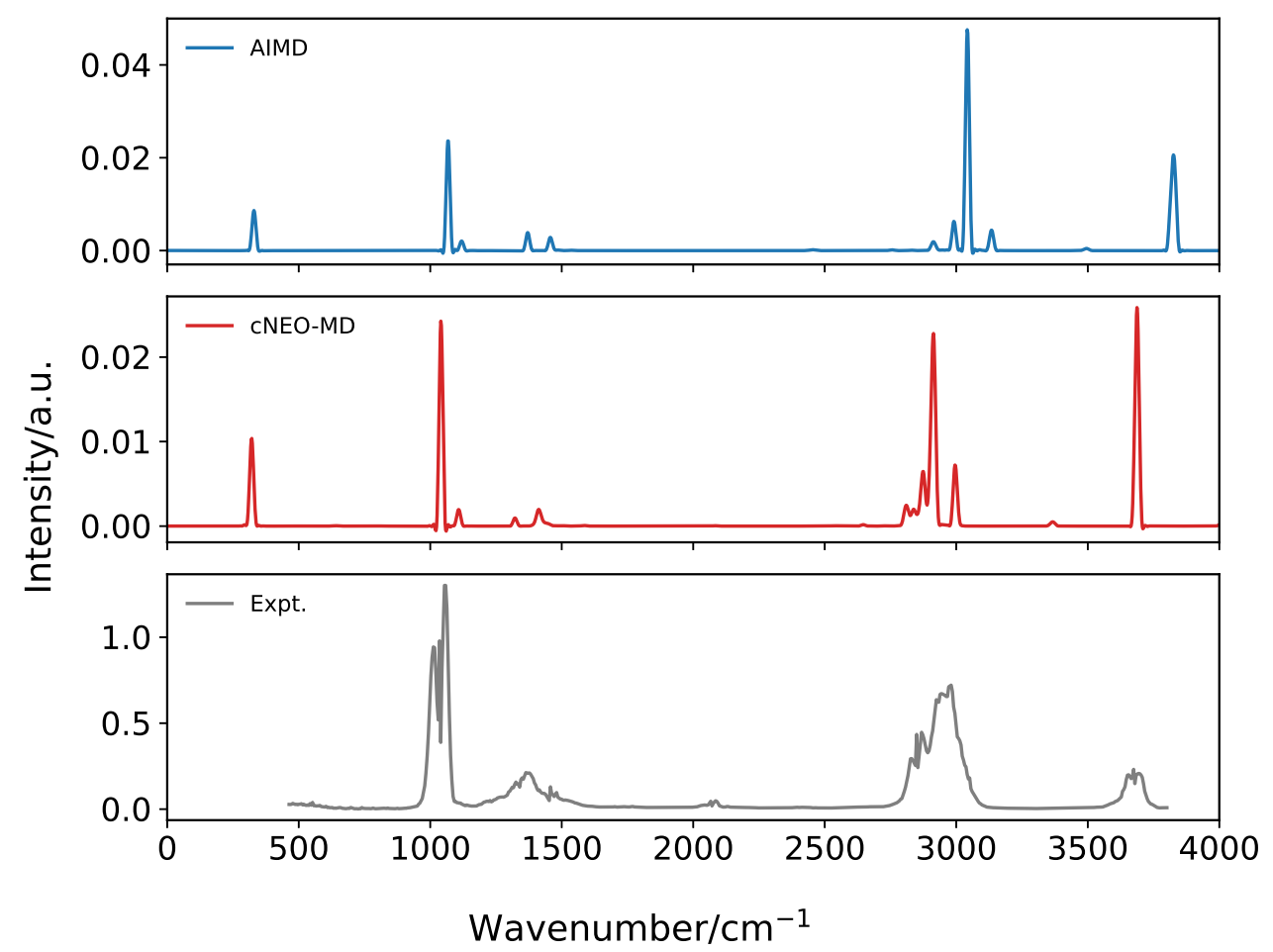

(b) IR spectra

Figure 3: Power spectra and IR spectra of a single $\mathrm{CH}_{3} \mathrm{OH}$ molecule by AIMD and cNEO$\mathrm{MD}$ at $300 \mathrm{~K}$. Experimental vibrational frequencies shown as dashed vertical lines in (a) and experimental spectra in (b) are both from the National Institute of Standards and Technology (NIST) websites. 
dependence. We choose a single $\mathrm{CH}_{3} \mathrm{OH}$ molecule and performed both AIMD and cNEO-MD at $10 \mathrm{~K}, 100 \mathrm{~K}, 300 \mathrm{~K}$, and $1000 \mathrm{~K}$. The resulting IR spectra are presented in Fig. 4. We can see that the effect of temperature on AIMD and cNEO-MD are similar, and they both display a slight redshift of vibrational frequencies and broadening of peaks as the temperature increases. However, these effects are relatively small for both AIMD and cNEO-MD simulations if the system is at or below room temperature. This is because at low temperatures, molecular dynamics still mainly samples the region near the equilibrium position, which is mostly harmonic. Therefore, the frequencies from both AIMD and cNEO-MD are close to their corresponding values from harmonic Hessian calculations. (see Table S3) This is no longer true as the temperature increases to $1000 \mathrm{~K}$, where most peaks become smeared and it becomes difficult to assign peak positions.

In summary, we combined our recently developed methods of cNEO-DFT and CMESMD and created a new method - cNEO-MD, that can accurately and efficiently describe nuclear quantum effects in molecular simulations. With cNEO-MD, we obtained power and IR spectra for water, formic acid, and methanol molecules. Compared with AIMD, cNEO-MD gives vibrational spectra that are in much better agreement with experimental results, especially for the stretch modes involving hydrogen atoms. Isotope effects on the vibrational spectra can also be accurately described by cNEO-MD, and subtle structures in the spectra such as Fermi resonances can also be accurately described provided that the electronic density functional approximation is reasonably accurate. Furthermore, since cNEO-DFT has the same formal scaling as conventional DFT, cNEO-MD is an affordable computational method for incorporating nuclear quantum effects into MD simulations. This work opens the possibility of using cNEO-MD in the study of static and dynamic properties of chemical and biological systems with significant nuclear quantum effects. 


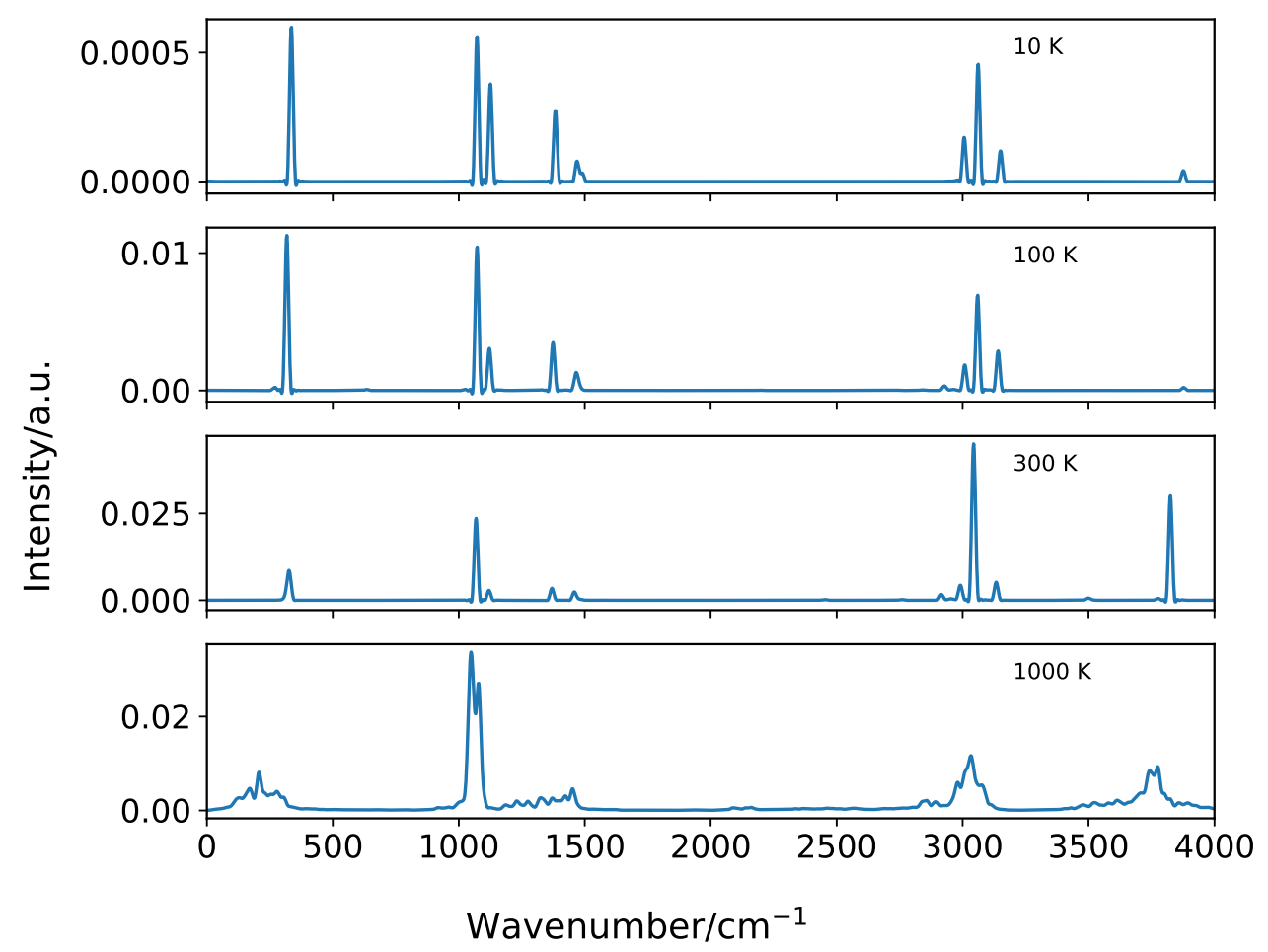

(a) AIMD

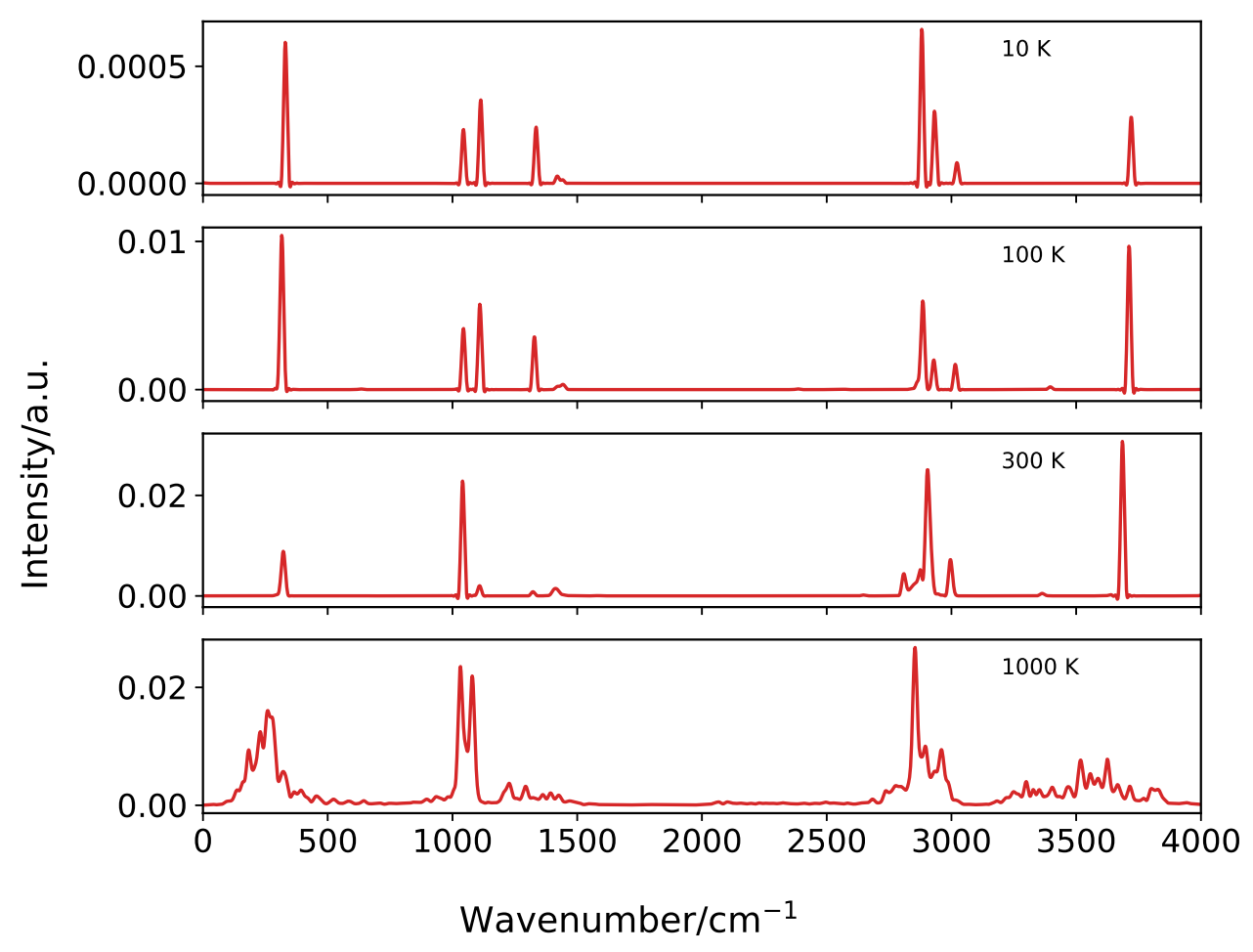

(b) cNEO-MD

Figure 4: IR spectra of a single $\mathrm{CH}_{3} \mathrm{OH}$ molecule by (a) AIMD and (b) cNEO-MD at different temperatures 


\section{Supporting Information Available}

The following files are available free of charge. See Supplementary Information for the PB4-D basis set test for proton and deuterium, the comparison of harmonic vibrational frequencies by CCSD $(\mathrm{T})$ and DFT with different functionals, power spectra and IR spectra by different $N V E$ trajectories, and molecular vibrational frequencies by MD simulations and Hessian calculations.

\section{Acknowledgement}

We are grateful for the support and funding from the University of Wisconsin via the Wisconsin Alumni Research Foundation. We also thank Dr. Huan Wang and Mr. James Langford for helpful discussions.

\section{References}

(1) Tuckerman, M. E.; Martyna, G. J. J. Phys. Chem. B 2000, 104, 159-178.

(2) Tuckerman, M. E. J. Phys.: Condens. Matter 2002, 14, R1297-R1355.

(3) Iftimie, R.; Minary, P.; Tuckerman, M. E. PNAS 2005, 102, 6654-6659.

(4) Morrone, J. A.; Car, R. Phys. Rev. Lett. 2008, 101, 017801.

(5) McBride, C.; Noya, E. G.; Aragones, J. L.; Conde, M. M.; Vega, C. Phys. Chem. Chem. Phys. 2012, 14, 10140-10146.

(6) Ceriotti, M.; Cuny, J.; Parrinello, M.; Manolopoulos, D. E. PNAS 2013, 110, 1559115596.

(7) Marsalek, O.; Markland, T. E. J. Phys. Chem. Lett. 2017, 8, 1545-1551.

(8) Miller, W. H. J. Phys. Chem. A 2001, 105, 2942-2955. 
(9) Meyer, H.-D.; Worth, G. A. Theor Chem Acc 2003, 109, 251-267.

(10) Markland, T. E.; Ceriotti, M. Nat Rev Chem 2018, 2, 1-14.

(11) Beck, M. H.; Jäckle, A.; Worth, G. A.; Meyer, H. D. Phys. Rep. 2000, 324, 1-105.

(12) Feynman, R.; Hibbs, A. Quantum mechanics and path integrals; McGraw-Hill: New York, 1965.

(13) Chandler, D.; Wolynes, P. G. J. Chem. Phys. 1981, 74, 4078-4095.

(14) Cao, J.; Voth, G. A. J. Chem. Phys. 1994, 100, 5106-5117.

(15) Craig, I. R.; Manolopoulos, D. E. J. Chem. Phys. 2004, 121, 3368-3373.

(16) Hone, T. D.; Rossky, P. J.; Voth, G. A. J. Chem. Phys. 2006, 124, 154103.

(17) Rossi, M.; Ceriotti, M.; Manolopoulos, D. E. J. Chem. Phys. 2014, 140, 234116.

(18) Herrero, C. P.; Ramírez, R. J. Phys.: Condens. Matter 2014, 26, 233201.

(19) Ceriotti, M.; Fang, W.; Kusalik, P. G.; McKenzie, R. H.; Michaelides, A.; Morales, M. A.; Markland, T. E. Chem. Rev. 2016, 116, 7529-7550.

(20) Habershon, S.; Fanourgakis, G. S.; Manolopoulos, D. E. J. Chem. Phys. 2008, 129, 074501 .

(21) Witt, A.; Ivanov, S. D.; Shiga, M.; Forbert, H.; Marx, D. J. Chem. Phys. 2009, 130, 194510.

(22) Ivanov, S. D.; Witt, A.; Shiga, M.; Marx, D. J. Chem. Phys. 2010, 132, 031101.

(23) Xu, X.; Yang, Y. J. Chem. Phys. 2020, 152, 084107.

(24) Xu, X.; Yang, Y. J. Chem. Phys. 2020, 153, 074106.

(25) Capitani, J. F.; Nalewajski, R. F.; Parr, R. G. J. Chem. Phys. 1982, 76, 568-573. 
(26) Kreibich, T.; Gross, E. K. U. Phys. Rev. Lett. 2001, 86, 2984-2987.

(27) Bochevarov, A. D.; Valeev, E. F.; Sherrill, C. D. Mol. Phys. 2004, 102, 111-123.

(28) Nakai, H. Int. J. Quantum Chem. 2007, 10\%, 2849-2869.

(29) Ishimoto, T.; Tachikawa, M.; Nagashima, U. Int. J. Quantum Chem. 2009, 109, 26772694.

(30) Pavošević, F.; Culpitt, T.; Hammes-Schiffer, S. Chem. Rev. 2020, 120, 4222-4253.

(31) Xu, X.; Yang, Y. J. Chem. Phys. 2021, 154, 244110.

(32) Barone, V. J. Chem. Phys. 2004, 120, 3059-3065.

(33) Barone, V. J. Chem. Phys. 2005, 122, 014108.

(34) Barone, V.; Biczysko, M.; Bloino, J. Phys. Chem. Chem. Phys. 2014, 16, 1759-1787.

(35) Yang, Y.; Brorsen, K. R.; Culpitt, T.; Pak, M. V.; Hammes-Schiffer, S. J. Chem. Phys. $2017,147,114113$.

(36) Brorsen, K. R.; Yang, Y.; Hammes-Schiffer, S. J. Phys. Chem. Lett. 2017, 8, 3488-3493.

(37) Chen, Z.; Yang, Y. arXiv:2111.12832 [physics] 2021, arXiv: 2111.12832.

(38) Sun, Q.; Berkelbach, T. C.; Blunt, N. S.; Booth, G. H.; Guo, S.; Li, Z.; Liu, J.; McClain, J. D.; Sayfutyarova, E. R.; Sharma, S.; Wouters, S.; Chan, G. K.-L. WIREs Comput Mol Sci 2018, 8, e1340.

(39) Sun, Q. et al. J. Chem. Phys. 2020, 153, 024109.

(40) Larsen, A. H. et al. J. Phys.: Condens. Matter 2017, 29, 273002.

(41) Dunning, T. H. J. Chem. Phys. 1989, 90, 1007-1023.

(42) Yu, Q.; Pavošević, F.; Hammes-Schiffer, S. J. Chem. Phys. 2020, 152, 244123. 
(43) Perdew, J. P.; Ernzerhof, M.; Burke, K. J. Chem. Phys. 1996, 105, 9982-9985.

(44) Raghavachari, K.; Trucks, G. W.; Pople, J. A.; Head-Gordon, M. Chem. Phys. Lett. $1989,157,479-483$.

(45) Nosé, S. J. Chem. Phys. 1984, 81, 511-519.

(46) Hoover, W. G. Phys. Rev. A 1985, 31, 1695-1697.

(47) Gordon, R. G. J. Chem. Phys. 1965, 43, 1307-1312.

(48) Berens, P. H.; Wilson, K. R. J. Chem. Phys. 1981, 74, 4872-4882.

(49) Wang, H.; Agmon, N. J. Phys. Chem. A 2016, 120, 3117-3135.

(50) Lamaire, A.; Wieme, J.; Rogge, S. M. J.; Waroquier, M.; Van Speybroeck, V. J. Chem. Phys. 2019, 150, 094503.

(51) Benedict, W. S.; Gailar, N.; Plyler, E. K. J. Chem. Phys. 1956, 24, 1139-1165.

(52) McCoy, A. B.; Silbert, E. L. J. Chem. Phys. 1990, 92, 1893-1901.

(53) Shiga, M.; Nakayama, A. Chem. Phys. Lett. 2008, 451, 175-181.

(54) Rossi, M.; Kapil, V.; Ceriotti, M. J. Chem. Phys. 2018, 148, 102301.

(55) Maçôas, E. M. S.; Lundell, J.; Pettersson, M.; Khriachtchev, L.; Fausto, R.; Räsänen, M. J. Mol. Spectrosc. 2003, 219, 70-80.

(56) Yu, Y.; Wang, Y.; Lin, K.; Hu, N.; Zhou, X.; Liu, S. J. Phys. Chem. A 2013, 117, $4377-4384$.

(57) Thomas, M.; Brehm, M.; Fligg, R.; Vöhringer, P.; Kirchner, B. Phys. Chem. Chem. Phys. 2013, 15, 6608-6622.

(58) Thomas, M. Theoretical Modeling of Vibrational Spectra in the Liquid Phase; Springer Theses; Springer International Publishing, 2017. 